\title{
Uso de bomba centrífuga no pós-operatório de cirurgia cardíaca
}

Paulo M. PÊGO-FERNANDES *, Luiz Felipe P. MOREIRA *, Luís Alberto DALLAN *, José Otávio C. AULER JÚNIOR *, Noedir A. G. STOLF *, Sérgio Almeida de OLIVEIRA *, Adib D. JATENE *.

PÊGO-FERNANDES, P. M.; MOREIRA, L. F. P.; DALLAN, L. A.; AULER JÚNIOR, J. O. C.; STOLF, N. A. G.; OLIVEIRA, S. A.; JATENE, A. D. - Uso de bomba centrífuga no pós-operatório de cirurgia cardíaca. Rev. Bras. Cir. Cardiovasc., 7(4):262-268, 1992.

RESUMO: Nos casos de choque cardiogênico após cirurgia cardíaca com auxílio da circulaçäo extracorpórea refratários às drogas e ao balāo intra-aórtico, as bombas centrífugas têm sido a primeira opçāo em vários Serviços. Esse fato deve-se à facilidade de manuseio, de instalaçāo, custo razoável, grande disponibilidade, e alto grau de eficiência. $O$ objetivo deste trabalho é o relato da experiência do Instituto do Coração com 8 pacientes submetidos a essa terapêutica, no período de maio de 1990 a dezembro de 1991 . Nesses 8 doentes foi utilizado previamente balāo intra-aórtico e feito uso maciço de drogas vasoativas. A idade variou de 54 a 66 anos. Três foram submetidos a revascularizaçāo do miocárdio, 2 a correçāo de aneurisma de ventrículo esquerdo, 2 a troca de valva mitral e 1 a transplante cardíaco. Em 7 a assistência foi de ventrículo esquerdo e em 1 de direito. A duração da assistência variou de 18 a 126 horas. Ocorreram três óbitos em assistência, sendo que dois eram pacientes em "ponte" para transplante que năo obtiveram doador, e um morreu por complicaçōes de sangramento e insuficiência renal aguda. Dos 5 (62,5\%) pacientes retirados da assistência, 2 faleceram tardiamente sendo $1 \mathrm{com}$ pulmảo de choque e $1 \mathrm{com}$ complicação neurológica e insuficiência renal. Quando comparamos a evoluçảo clínica com o pico de CKMB, verificamos que os 3 pacientes com pico maior que 80 faleceram, 2 em assistência e 1 tardiamente. Dos 5 doentes com pico de CKMB menor que 80,4 foram retirados de assistência, com 3 sobreviventes tardios, e o único que morreu em assistência foi por problemas de sangramento. Os $3(35 \%)$ pacientes sobreviventes estāo no $5^{\circ}, 9^{\circ}$ e $19^{\circ}$ meses de seguimento, 2 em classe funcional II e 1 em classe l. A utilização precoce, antes de complicaçōes irreversíveis, da assistência circulatória, deverá permitir resultados progressivamente melhores, nesse grupo de pacientes de alto risco.

DESCRITORES: bomba centrífuga, mecânica, em cirurgia cardíaca; circulaçāo assistida.

\section{INTRODUÇÃO}

Choque cardiogênico é uma causa importante de morte em pacientes de alto risco no pós-operatório de cirurgia cardíaca. Terapia médica convencional e balăo intra-aórtico proporcionam suporte circulatório adequado e sobrevida em aproximadamente metade dos pacientes que não podem ser retirados de circulação extracorpórea após uma cirurgia cardíaca tecnicamente bem sucedida ${ }^{4.12}$. Quando esses métodos falham, é necessária uma abordagem mais agressiva para o suporte tempo- rário da função ventricular com a subseqüente manutenção da circulação sistêmica e/ou pulmonar, assim como para diminuir o trabalho dos ventrículos deprimidos. Na maior parte das séries relatadas, a necessidade de suporte ventricular temporário atinge $1 \%$ dos pacientes adultos submetidos a cirurgia cardíaca com proteção miocárdica 5 , 10, 13.

No Instituto do Coração no período de maio de 1990 a dezembro de 1991, submetemos 8 pacientes a circulação assistida com bomba centrífuga após cirurgia cardíaca, correspondendo a $0,3 \%$ dos adul-

Trabalho realizado no Instituto do Coração do Hospital das Clínicas da Faculdade de Medicina da Universidade de São Paulo. São Paulo, SP, Brasil. Apresentado ao $19^{\circ}$ Congresso Nacional de Cirurgia Cardíaca, Sáo Paulo, SP, 7 a 9 de maio, 1992.

* Do Instituto do Coração do Hospital das Clínicas da Faculdade de Medicina da Universidade de Sâo Paulo.

Endereço para separatas: Paulo Pègo-Fernandes, Av. Dr. Enéas de Carvalho Aguiar, 44. Divisão Cirúrgica. 05403 São Paulo, SP. Brasil. 
PÊGO-FERNANDES, P. M.; MOREIRA, L. F. P.; DALLAN, L. A.; AULER JÚNIOR, J. O. C.; STOLF, N. A. G.; OLIVEIRA, S. A.; JATENE, A. D. - Uso de bomba centrífuga no pós-operatório de cirurgia cardíaca. Rev. Bras. Cir. Cardiovasc., 7(4):263-268, 1992.

tos operados nesse período. O objetivo deste trabalho é transmitir os dados que utilizamos nesses pacientes e as liçōes aprendidas nesse período.

\section{CASUÍSTICA E MÉTODOS}

Após utilização adequada de drogas vasoativas e de balāo intra-aórtico, temos adotado como protocolo para o uso de bomba centrífuga, os seguintes parâmetros hemodinâmicos:

a) falência ventricular esquerda definida como:

1. pressão capilar pulmonar $>20 \mathrm{~mm} \mathrm{Hg}$,

2. pressão arterial sistólica $<10 \mathrm{~mm} \mathrm{Hg}$,

3. índice cardíaco < 2 litros/minuto/metro quadrado de superfície corpórea $\left(1 / \mathrm{min} / \mathrm{m}^{2}\right)$.

b) falência ventricular direita definida como:

1. pressão atrial direita $(P A D)>20 \mathrm{~mm} \mathrm{hg}$,

2. pressão capilar pulmonar $(\mathrm{PCP})<10 \mathrm{~mm}$ $\mathrm{Hg}$, e/ou

3. $P A D-P C P>10 \mathrm{~mm} \mathrm{Hg}$,

4. índice cardíaco $<21 / \mathrm{min} / \mathrm{m}^{2}$ )

c) falência ventricular presente após correçāo de

1. gasometria arterial e eletrólitos;

2. hipovolemia;

3. hipervolemia;

4. arritmias;

5. hipotermia;

6. sangramento cirúrgico.

A essas condiçōes deve se agregar o fato de a cirurgia cardíaca ter sido tecnicamente bem sucedida, com correção total da afecção anatômica. Nos casos em que a falência ventricular se afigure como irreversível, o paciente necessita preencher os critérios de receptor para eventual transplante cardíaco, e entraria num segundo grupo de doentes, os pacientes em que a bomba centrífuga é utilizada como "ponte" para transplante. Contra-indicaçōes para a assistência circulatória sāo as falências orgânicas que inviabilizem o prognóstico do paciente, como falência neurológica, pulmonar, renal ou mesentérica irreversíveis; endocardite bacteriana; disfunçāo hepática ou discrasias sangüíneas severas.

Durante a assistência ventricular esquerda mantemos um fluxo sangüíneo total sistêmico (débito do ventrículo nativo + fluxo da bomba centrífuga) acima de $2,21 / \mathrm{min} / \mathrm{m}^{2}$. A pressão capilar pulmonar é mantida entre 5 e $15 \mathrm{~mm} \mathrm{Hg}$. A pressão venosa central deve ser mantida até $15 \mathrm{~mm} \mathrm{Hg}$; caso esteja acima de $20-25 \mathrm{~mm} \mathrm{Hg}$ deve ser pensada a possibilidade de assistência ventricular direita, especialmente se não se consegue manter fluxo pela bomba de assistência esquerda (por falta de retorno sangüíneo). Para a assistência ventricular direita, medidas hemodinâmicas similares são seguidas.

Nas primeiras horas de circulação assistida não utilizamos heparina. Passadas as primeiras horas $e$ não havendo sangramento importante, iniciamos heparinização endovenosa contínua, que é controlada pela realização a cada 4 horas do tempo de coagulação ativado (TCA). Mantemos o TCA entre 150 e 200 segundos. Realizamos coagulograma a cada 12 horas.

Tendo em vista que esses pacientes são muito manuseados, e que a incisāo cirúrgica não é totalmente fechada, eles são colocados em ambiente utilizado para doentes transplantados e tomamos os cuidados de assepsia e anti-sepsia semelhantes. A antibioticoterapia é semelhante à dos transplantados, atualmente cefalosporina de $2^{\mathrm{a}}$ geração, associada a aminoglicosídeo.

Os controles gerais de enfermagem são feitos a cada hora, as medidas de débito cardíaco a cada 4 horas. $O$ ecocardiograma transesofágico foi utilizado durante o período de assistência, para avaliaçāo da evoluçăo da função ventricular esquerda. Os demais exames seguem a periodicidade habitual de pacientes graves.

A bomba centrífuga utilizada foi a da Biomedics, que é um dispositivo dirigido eletromecanicamente. A bomba consiste em um cone de acrílico no qual estão três cones magnéticos acoplados e rotatórios, e o sangue é movido através de força centrífuga. Esse dispositivo pode prover um fluxo de até 6 a 8 litros/minuto.

Foram tratados 8 pacientes. A idade variou de 54 a 66 anos. Três foram submetidos a revascularizaçāo do miocárdio, 2 a correção de aneurisma de ventrículo esquerdo, 2 a troca de valva mitral e 1 a transplante cardíaco (Gráfico 1). Em sete a assistência foi de ventrículo esquerdo; no paciente submetido a transplante cardíaco assistiu-

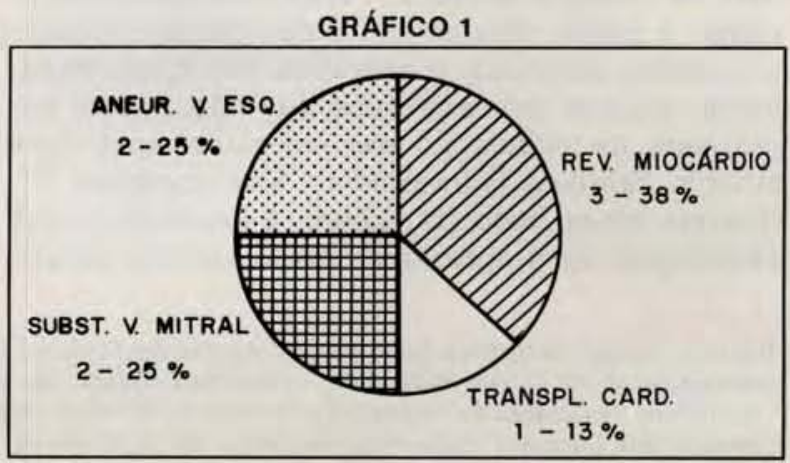


PÊGO-FERNANDES, P. M.; MOREIRA, L. F. P.; DALLAN, L. A.; AULER JÚNIOR, J. O. C.; STOLF, N. A. G.; OLIVEIRA, S. A.; JATENE, A. D. - Uso de bomba centrífuga no pós-operatório de cirurgia cardíaca. Rev. Bras. Cir. Cardiovasc., 7(4):263-268, 1992.

se o ventrículo direito. A duração da assistência variou de 18 a 126 horas. Dois pacientes que haviam sido revascularizados apresentaram infarto agudo do miocárdio (IAM) maciço e a indicação da assistência foi como "ponte" para transplante cardíaco. Um paciente era bronquítico severo e outro apresentava graves distúrbios de coagulação. Sete pacientes não "saíram" de extracorpórea (CEC) e o oitavo apresentou parada cardíaca no $2^{\circ}$ dia de pósoperatório por oclusāo de ponte de safena com IAM anterior extenso; reaberto, recolocado em CEC e utilizada circulação assistida como "ponte".

Todos os pacientes foram "fechados" com compressas para reforço da hemostasia, as cânulas utilizadas foram as mesmas usadas em CEC e exteriorizadas pela extremidade superior da incisāo, e somente a pele foi suturada. A assistência ventricular esquerda foi com canulação através da aurícula esquerda e raiz da aorta. A assistência direita foi feita pela canulação através da aurícula direita e do tronco pulmonar.

Para decidirmos pela descontinuidade da assistência circulatória, temos utilizado metodologia semelhante à de MYERS et alii ${ }^{5}$ associada ao emprego de ecocardiograma transesofágico ${ }^{8}$. Com isso associamos os dados hemodinâmicos, que passaremos a referir, com a visibilizaçāo da funçāo ventricular. Normalmente descontinuamos o uso da bomba de $12 / 12$ horas, por um período de 60 segundos, com o intuito de observarmos uma evolução seqüencial da funçāo ventricular. Quando o ventrículo acometido é capaz de manter uma pressão atrial menor do que $20 \mathrm{~mm} \mathrm{Hg}$, pressão sistólica aórtica maior que $100 \mathrm{~mm} \mathrm{Hg}$, e índice cardíaco maior que $21 / \mathrm{min} / \mathrm{m}^{2} \mathrm{com}$ a bomba desligada por 60 segundos, a assistência pela bomba é progressivamente diminuída a cada 6 horas para permitir ao ventrículo assistido uma readaptaçăo gradual.
Quando o fluxo pela bomba centrífuga está próximo a $11 / \mathrm{min}$, aplicamos heparinização de $4 \mathrm{mg} / \mathrm{kg}$; nunca mantemos o fluxo menor do que $0,51 / \mathrm{min}$.

\section{RESULTADOS}

A duraçăo da assistência variou de 18 a 126 horas. Ocorreram três óbitos em assistência, sendo que dois eram pacientes em "ponte" para transplante, que não obtiveram doador, e um morreu por complicaçōes de sangramento e insuficiência renal aguda. Dos 5 pacientes retirados da assistência, 2 faleceram tardiamente, sendo $1 \mathrm{com}$ pulmão de choque e $1 \mathrm{com}$ complicação neurológica e insuficiência renal.

Analisando a afecção de base com a evoluçāo clínica, notamos que os 3 pacientes revascularizadores morreram, 2 em assistência devido a infarto maciço e 1 por complicaçōes devido a diátese hemorrágica. Dos 2 pacientes submetidos a troca de valva mitral, 1 sobreviveu e 1 morreu por sangramento e insuficiência renal. Os 2 doentes submetidos a correção de aneurisma de ventrículo esquerdo sobreviveram à assistência, 1 morrendo tardiamente por complicaçōes neurológicas, com boa função hemodinâmica - mostrando que, após o período de adaptação a uma menor complacência ventricular, esses pacientes se recuperam bem. $O$ doente submetido a trasplante cardíaco, que necessitou de assistência de ventrículo direito por hipertensāo pulmonar, também se recuperou bem após adaptação desse ventrículo (Gráfico 2).

Quando comparamos a evolução clínica com o pico de CKMB, verificamos que os 3 pacientes com pico maior que 80 faleceram, 2 em assistência e 1 tardiamente. Dos 5 doentes com pico de CKMB menor que 80,4 foram retirados de assistência,
GRÁFICO 2

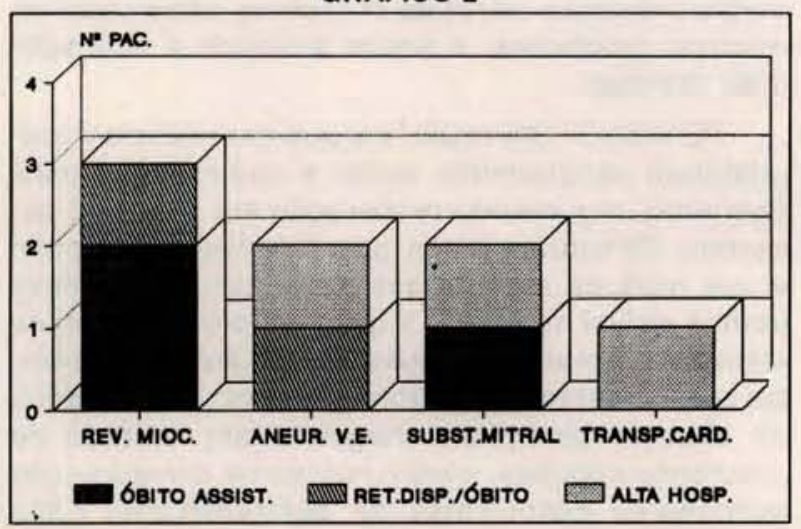

GRÁFICO 3

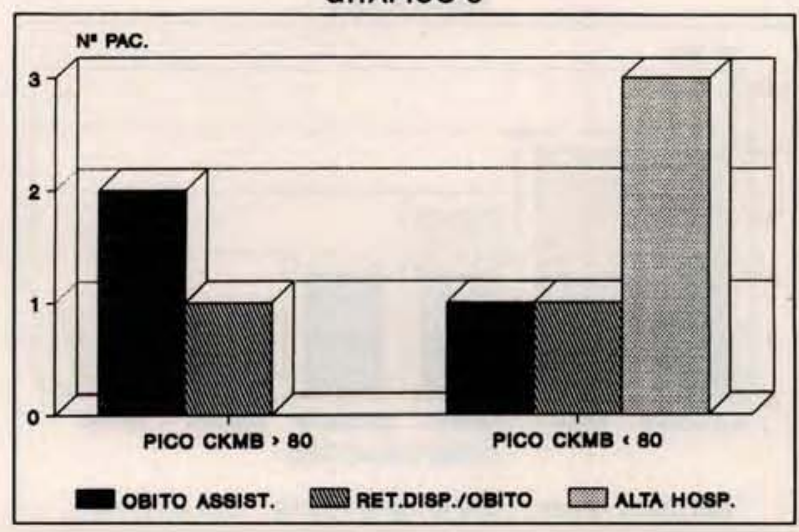


PÊGO-FERNANDES, P. M.; MOREIRA, L. F. P.; DALLAN, L. A.; AULER JÚNIOR, J. O. C.; STOLF, N. A. G.; OLIVEIRA, S. A.; JATENE, A. D. - Uso de bomba centrífuga no pós-operatório de cirurgia cardíaca. Rev. Bras. Cir. Cardiovasc., 7(4):263-268, 1992.

com 3 sobreviventes tardios, e o único que morreu em assistência foi por problemas de sangramento (Gráfico 3).

Dois pacientes apresentaram sangramento severo: 1 já era portador de diátese hemorrágica. Nesses 2 doentes a hemorragia foi o fator que levou ou precipitou o óbito. Mantendo o nosso controle de heparinização com TCA entre 150 e 200 segundos, nāo tivemos nenhum problema de trombose ou embolia. Um paciente apresentou embolia pulmonar maciça e óbito após suspensāo da heparina para retirada de compressas (Gráfico 4). Após a retirada das compressas e fechamento completo da incisāo, nāo notamos complicaçōes infecciosas em nenhum paciente. Quando as plaquetas ficaram abaixo de $50000 / \mathrm{mm}$, foram ministrados concentrados.

Os 5 pacientes retirados de assistência necessitaram de uma permanência maior na terapia intensiva, com cuidados renais e pulmonares. Notamos que todos apresentaram aumento de uréia e creatinina, provavelmente por transfusāo de hemoderivados, perfusāo renal diminuida e componente de fluxo circulatório nāo pulsátil.

Os três sobreviventes estāo no $5^{\circ}, 9^{\circ}$ e $19^{\circ}$ meses de seguimento, 2 em classe funcional II e 1 em classe I.

\section{COMENTÁRIOS}

Para termos uma idéia do potencial da necessidade da assistência circulatória em cirurgia cardiaca, consideremos que um bilhāo de pessoas vivam nos Estados Unidos, Japão, Europa e América Latina. Teremos um milhão de potenciais pacientes de cirurgia cardíaca por ano (essa taxa de 100 por milhão de pacientes cirúrgicos não é verdadeira nos Estados Unidos, e é aproximada na Europa), e,

\section{GRÁFICO 4}

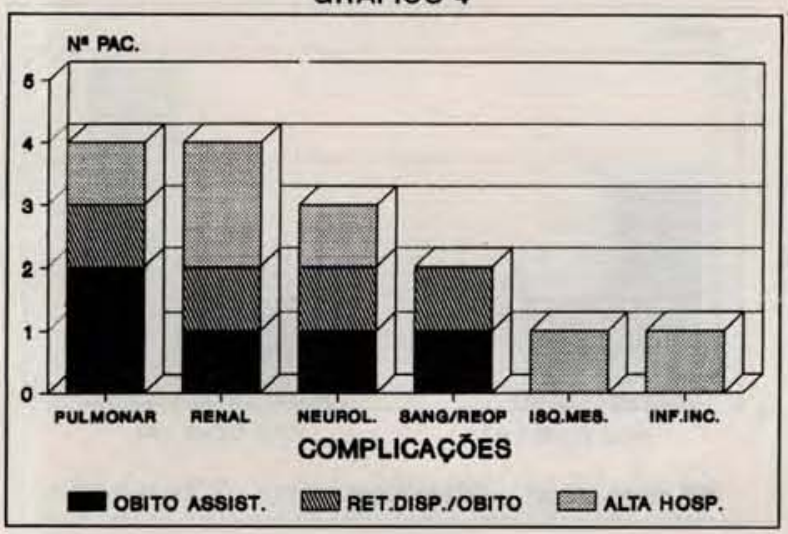

desses, aproximadamente 850000 sāo adultos. A incidência de falência cardíaca após cirurgia cardíaca caiu de $10 \%$ em 1975 para $1 \%$ atualmente. Logo, existem potencialmente 8500 doentes por ano submetidos a cirurgia cardíaca, que se beneficiaram de algum tipo de assistência circulatória ${ }^{13}$.

Podemos considerar seis categorias básicas de assistência:

1. Assistência ventricular mecânica em série, como a contrapulsaçāo com balāo intra-aórtico e o patch aórtico dinâmico.

2. Assistência ventricular mecânica em paralelo dada pelos vários dispositivos de assistência como as bombas centrífugas.

3. Substituiçăo mecânica: coração artificial total para substituiçāo definitiva ou provisória.

4. Pontes: dispositivos 2 ou 3 usados em pacientes que aguardam transplante cardíaco ou cardiopulmonar.

5. Substituiçōes biológicas como o transplante cardíaco ou cardiopulmonar.

6. Assistência biomecânica como a cardiomioplastia.

Neste trabalho tratamos dos tópicos 1,2 e 4 . Temos sempre utilizado o balāo intra-aórtico inicialmente, tanto pelo fato de considerarmos que, agindo assim, evitamos muitas vezes o uso de bombas centrífugas, como para proporcionar um componente pulsátil no período inicial de circulação assistida, quando o fluxo pelo ventrículo nativo é muito pequeno. Em 2 dos 8 pacientes a assistência circulatória foi utilizada como "ponte", fato anteriormente já comentado.

A circulação extracorpórea (CEC) prolongada antes do início da assistência ventricular significa problemas subseqüentes. A maioria dos investigadores comenta que as seqüelas de um período prolongado de CEC, especificamente níveis elevados de hemoglobina plasmática e diátese hemorrágica secundária a danos de componentes sangüíneos, reduz significamente a sobrevida com assistência ventricular, apesar de nāo ser uma contra-indicaçăo absoluta ${ }^{10}$. Temos observado os mesmos problemas, e temos tentando a indicação mais precoce.

PENNINGTON et alii ${ }^{9} \mathrm{em}$ sua experiência inicial relataram sangramento como a complicaçāo mais freqüente, requerendo reoperaçāo em 14 de 16 pacientes. Relataram ainda que hemólise foi comum e que mais da metade dos pacientes apresentava trombo visível no vórtex, e que um deles apresentou embolia sistêmica. Em nossa experiência 2 pacientes apresentaram sangramento severo: 1 necessitou de duas reoperaçōes, chegou a ser retirado de circulaçāo assistida, porém morreu de complicaçōes pulmonares decorrentes de politransfusāo. Esse 
PÊGO-FERNANDES, P. M.; MOREIRA, L. F. P.; DALLAN, L. A.; AULER JÚNIOR, J. O. C.; STOLF, N. A. G.; OLIVEIRA, S. A.; JATENE, A. D. - Uso de bomba centrífuga no pós-operatório de cirurgia cardíaca. Rev. Bras. Cir. Cardiovasc., 7(4):263-268, 1992.

paciente já apresentava diátese hemorrágica antes de ser colocado em assistência, porém mesmo assim foi instalada bomba centrífuga por ser paciente jovem, sem outros acometimentos. O paciente que apresentou parada cardíaca na terapia intensiva, por oclusão de ponte de safena para o ramo interventricular anterior no $2^{\circ}$ dia de pós-operatório, foi colocado em assistência circulatória como "ponte" para transplante cardíaco. No $4^{\circ}$ dia começou a apresentar febre e leucocitose, que relacionamos com as compressas. Com receio de sangramento à manipulaçāo, suspendemos a infusāo de heparina e acreditamos que essa tenha sido a causa da embolia pulmonar maciça que o levou a óbito no dia seguinte. Em estudo recentemente realizado por nós ${ }^{7}$, năo notamos hemólise importante quando esses dispositivos sāo usados por pouco tempo, em CEC habitual; porém neste grupo de 8 pacientes em que o uso é prolongado, também temos constatado hemólise e consumo de elementos figurados.

MAGOVERN et alii ${ }^{3}$ relataram assistência átrio esquerdo-aorta em 21 pacientes, dos quais 10 (48\%) foram retirados e $5(24 \%)$ tiveram alta hospitalar.
Todos estavam vivos com 1 a 3 anos de seguimento pós-operatório. Outros grupos ${ }^{1,2,6,11}$ relataram resultados semelhantes. Dos nossos 8 pacientes, 5 $(62,5 \%)$ foram retirados da assistência e 3 (35\%) receberam alta hospitalar. Em todas essas experiências ${ }^{1-3,6.11}$ os pacientes sobreviventes estāo assintomáticos ou pouco sintomáticos em relação à insuficiência cardíaca, fato também observado por nós.

Por se tratar de método de alto custo, devido a hemoderivados, exames laboratoriais, cuidados de isolamento, balão intra-aórtico, dispositivo descartável da bomba centrifuga, com sobrecarga de trabalho para equipe médica e paramédica, acreditamos que devamos nos esforçar para não abrirmos muitas exceçōes ao protocolo de indicaçōes e contra-indicaçōes, sob pena de inviabilizarmos o procedimento para os pacientes que realmente tenham oportunidade de serem beneficiados pela circulaçāo assistida. Esse rigor, paradoxalmente, nos deverá levar a indicaçōes mais precoces da assistência circulatória, numa fase clínica em que o acometimento de outros órgảos é pequeno e, portanto, melhor prognóstico.

RBCCV 44205-188

PÊGO-FERNANDES, P. M.; MOREIRA, L. F. P.; DALLAN, L. A.; AULER JÚNIOR, J. O.C.; STOLF, N. A. G.; OLIVEIRA, S. A.; JATENE, A. D. - Assisted circulation in cardiac surgery. Rev. Bras. Cir. Cardiovasc., $7(4): 263-268,1992$.

ABSTRACT: In patients with cardiogenic shock following cardiopulmonary bypass, not responsive to intraortic balloon pump and drugs, the centrifugal pumps are the first option in many centers. These centers that use modern vortex pumps cite their simplicity, availability, relatively low cost, and low amount of blood trauma. The aim of this study is to describe the experience of InCór with 8 patients submitted to assisted circulation with biopump from May 1990 to December 1991. Intraortic balloon counterpulsation was used in these 8 patients with conventional medical therapy. Their ages ranged from 54 to 66 . Three patients were submitted to coronary bypass, 2 to mitral valve replacement, 2 to left ventricular aneurysm correction and 1 to heart transplantation. Left atrial to aortic bypass was done in 7 patients and in 1 , right atrial to pulmonary bypass. The patients were assisted from 18 to 126 hours. Three patients died during assisted circulation: 2 were in bridge to transplantation and didn't achieve donor organ, and 1 died due to bleeding and acute renal failure. Five (62.5\%) patients were weaned from the device, but 2 died at hospital, 1 due to respiratory failure and 1 due to neurologic and renal failure. When we compared the clinical evaluation with CKMB, we noted that 3 patients with CKMB greater than 80 died, 2 during assisted circulation. In 5 patients with CKMB less than 80,4 were weaned from the device and 3 were discharged from the hospital. Two patients discharged from the hospital are in NYHA functional class II and 1 in class I. The early utilization of assisted circulation will lead to better results in this group of high risk patients.

DESCRIPTORS: biopump, mechanic, in cardiac surgery; assisted circulation. 
PÊGO-FERNANDES, P. M.; MOREIRA, L. F. P.; DALLAN, L. A.; AULER JÚNIOR, J. O. C.; STOLF, N. A. G.; OLIVEIRA, S. A.; JATENE, A. D. - Uso de bomba centrífuga no pós-operatório de cirurgia cardíaca. Rev. Bras. Cir. Cardiovasc., 7(4):263-268, 1992.

\section{REFERÊNCIAS BIBLIOGRÁFICAS}

1 GOLDING, L. R.; JACOBS, G.; GROVES, L. K.; GILL, C. C.; NOSE, Y.; LOOP, F. D. - Clinical results of mechanical support of the failing left ventricle. J. Thorac. Cardiovasc. Surg., 83: 596-601, 1982.

2 GOLDING, L. A. R.; STEWART, R. W.; LOOP, F. D. Centrifugal pumps in clinical practice. In: UNGER, $F$ (ed.) Assisted circulation. 3. ed. Berlin-Heidelberg, Springer-Verlag, 1989. p. 160-166.

3 MAGOVERN, G. J.; PARK, S. B.; MAHER, T. D. - Use of a centrifugal pump without anticoagulants for postoperative left ventricular assist. World J. Surg., 9: 25-30, 1985.

4 McENANY, M. T.; KAY, H. R.; BUCKLEY, M. J.; DAGGETT, W. M.; ERDMANN, A. J.; MUNDTH, E. D.; RAO, R. S.; TOEUF; J. AUSTEN, G. - Clinical experience with intra-aortic balloon pump support in 728 patients. Circulation, 58 (Supl. 1): I 24, 1978.

5 MYERS, J. L.; PARR, G. V. S.; PAE Jr., W. E. - The role of the ventricular assist pump for postcardiotomy cardiogenic shock: four and one-half year experience. Artif. Organs, 5 (Supl.): 244-251, 1981.

6 PARK, S. B.; LISEBLER, G. A.; BURKHOLDER, J. A.; MAHER, T. D.; BENCKART, D. H.; MAGOVERN, G. J.; CHRISTLIEB, I. Y.; KAO, R. L. - Mechanical support of the failing heart. Ann. Thorac. Surg., 42: 627-631, 1986.

7 PÊGO-FERNANDES, P. M.; MIURA, F.; HIGA, S. S.; MOREIRA, L. F. P.; DALI AN, L. A.; CHAMONE, D. A. F.; OLIVEIRA, S. A.; STOLF, N. A. G.; JATENE,
A. D. - Hemólise em circulação extracorpórea: estudo comparativo entre bomba de rolete e bomba centrífuga. Rev. Bras. Cir. Cardiovasc., 4: 220-224, 1989.

8 PÊGO-FERNANDES, P. M.; MOREIRA, L. F. P.i JATENE, F. B.; AULER Jr., J. O. C.; MORAES, A. V.; JATENE, A. D. - Circulação assistida com bomba centrífuga de choque cardiogênico após cirurgia com extracorpórea Arq. Bras. Cardiol., 56: 313316, 1991.

9 PENNINGTON, D. G.; MERJAVY, J. P.; CODD, J. E. - Temporary mechanical support of patients with profounded ventricular failure. In: UNGER, F. (ed.): Assisted circulation II. New York, Springer Verlag, 1984. p. 85.

10 PIERCE, W. S. (Chairman) - Cardiac support. Trans. Am. Soc. Artif. Intern. Organs, 26: 625-631, 1980.

11 ROSE, D. M.; COLVIN, S. B.; CULLIFORD, A. T.; ISOM, D. W.; CUNNINGHAM Jr., J. N. - Late functional and hemodynamic status of surviving patients following insertion of the left heart assist device. J. Thorac. Cardiovasc. Surg., 86: 639-645, 1983.

12 SANFELIPPO, P. M.; BAKER, N. H.; EWY, G. H.; MOORE, P. J.; THOMAS, J. W.; BRAHOS, G. J.; McVICKER, R. F. - Experience with intra-aortic balloon counterpulsation. Ann. Thorac. Surg., 41: 36-41, 1986.

13 UNGER, F. - The present status of assisted circulation. In: UNGER, F. (ed.) Assisted circulation. 3. ed. Berlim-Heidelberg, Springer-Verlag, 1989. p. 1-4. 